NOTES AND STUDIES

\title{
THE OLD LATIN TEXTS OF THE MINOR PROPHETS. IV.
}

HAGGAI.

I.

Speculum. $\quad 6^{\circ}$ Seminastis multa et intulistis minima .

Cyprian. $9^{\circ}$

Haec dicit Dominus Omnipotens, eo quod domus mea deserta est, vos autem sectamini unusquisque 10 in domum suam, ${ }^{10}$ propterea abstinebit caelum a rore et terra subI trahet procreationes suas, ${ }^{11} \mathrm{et}$ inducam gladium super terram et super frumentum et super vinum et super oleum et super homines et super 12 pecora et super omnes labores manuum eorum. ${ }^{12}$ Et adivit Zorobabel filius Salathiel de tribu Iuda et Iesus filius Iosedech sacerdos magnus et omnes qui superfluerunt de populo vocis Domini Dei sui et verbum Aggaei prophetae, quoniam misit illum Dominus ad illos, et timuit plebs a facei Dei .

Speculum.

Auct. c. Fulg. II ${ }^{11}$ II.

instat in medio vestrum Donat.

I 6 Spec. lix Testim. iii 20
I 9-I I Cypr. Ad Demet. vi; Spec. lix II 5 Spec. iii
II II-I 4 Auct. contra Fulg. Donat. xvi

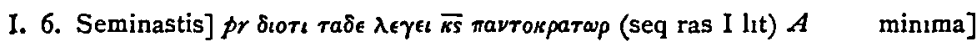

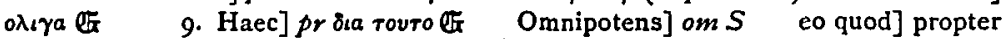
quod $S$ deserta est] $p r$ os $\aleph^{c .}$ (b? $\left.{ }^{\prime}\right)$ vid (rurs del) sectamini] satıs agitis $S$ ro. propterea abstinebit] ideo cessavit $S$ procreationes suas] fructus suos $S$

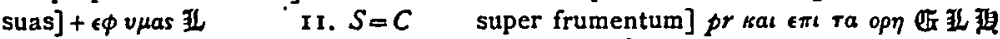

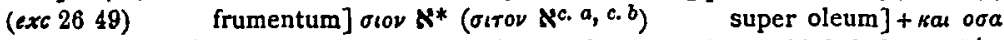

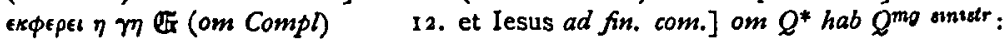

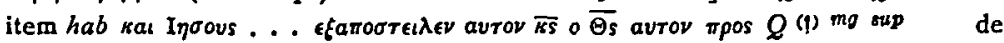

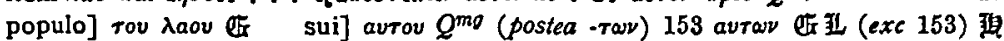

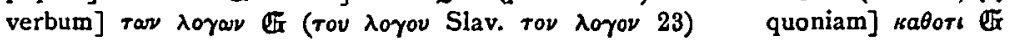
Dominus] +o Oeos avray [f]

Dei] Kvplov [ᄄ]

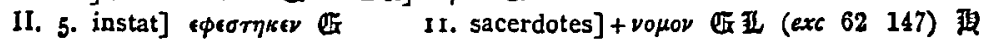


${ }_{12}{ }^{12} \mathrm{Si}$ alligaverit homo carnem sanctam in summo vestimento et tetigerit summitas vestimenti aliquam creaturam panis aut vini aut olei, si sanctificatur? Et responderunt sacerdotes et dixerunt : Non. ${ }_{13}{ }^{15} \mathrm{Et}$ dixit Dominus : Si tetigerit inquinatus in anima horum aliquid, I4 si inquinabitur? Et dixerunt sacerdotes: Inquinabitur. " $\mathrm{Et} \mathrm{dixit}$ Dominus : Si et populus hic, et sic gens ista, .

-..$\quad$ omnis qui illic accesserit inquinabitur

aI 21

22 mare et aridam . . . . ${ }^{22}$. . . . et convertam currus et sessores, et descendent equi et sessores eorum 23 unusquisque in gladio ad fratrem suum. ${ }^{29}$ In illo die, dicit Dominus omnipotens, accipiam te Zorobabel, filium Salathiel servum meum, et ponam te signaculum, quoniam te elegi, dicit Dominus omnipotens.

ZECHARIAH.

I.

${ }^{14}{ }^{14} \mathrm{Et}$ ait mihi angelus qui in me loquebatur . . . . . Tertullian.

I5 Zelatus sum Hierusalem et Sion zelo magno, ${ }^{15}$ et ira magna ego irascor Luc. Cal. super gentes quae se superponunt vobis; propter quod [Tyconius.] ego quidem iratus sum modice, ipsi autem adiecerunt in mala.

Tyconius.

II.

II I4 Coll. Carth. Gesta cclviii

II $21-23$ Tycon. Reg. Sept.

Zechariah. I $\mathrm{I}_{4}$ Tert. De came Christi xiv

Athan. i $36 \quad$ I 15 Tycon. Reg. Sept.

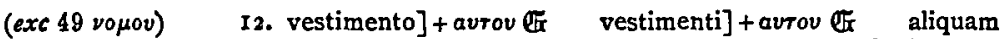

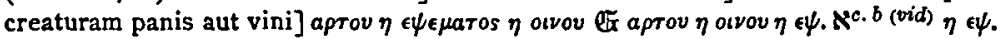

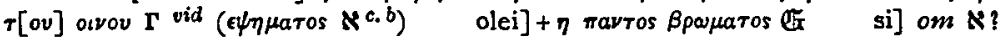

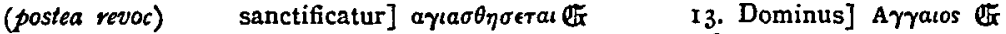

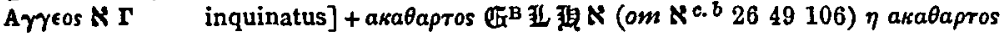

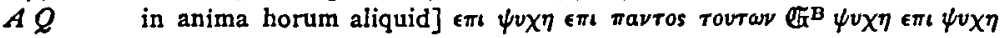

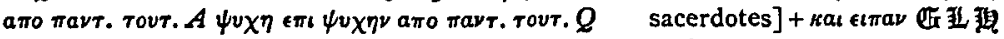

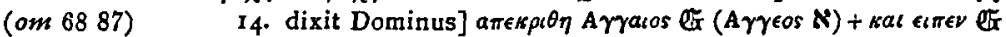
Si et] ourous [fic sic et $C_{c}$ hic] iste $C_{c}$ et sic gens ista] $C_{c}=F$ omnis...

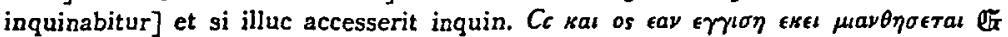

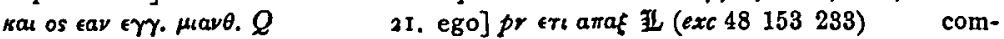

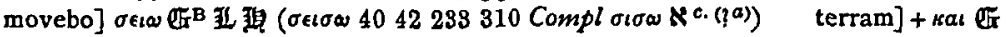

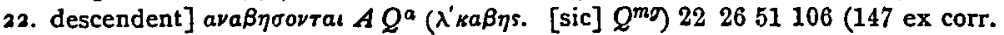

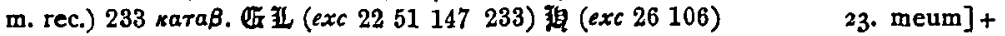

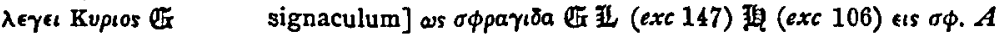
106147

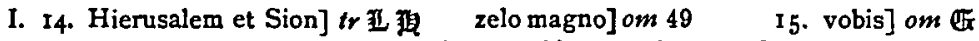

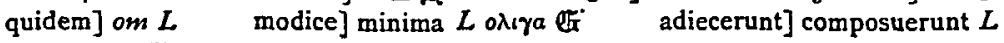

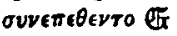


Speculum. $\quad 7,8{ }^{7}$ In Sion fugite qui conmoramini filiam Babylonis. ${ }^{8}$ Quia haec dicit Dominus omnipotens : post honorem misit me super gentes quae spoliaverunt vos, quia qui tangit vos sicut qui tangit pupillam oculi 9 ipsius; ${ }^{\circ}$ ecce ego iniciam manum meam super eos, et erunt in praedam his qui serviunt illis, et scient quoniam Dominus omnipotens misit me

Tyconius. ${ }^{\text {I } 3}{ }^{\text {13 }}$ Timeat a facie Domini omnis terra quoniam exsurrexit de nubibus sanctis.

Cyprian. III. I ${ }^{1}$ Et ostendit mihi Dominus Iesum sacerdotem illum magnum stantem ante faciem angeli Domini, et diabolus stabat ad dexteram Speculum. 2 eius adversari ei. ${ }^{2} \mathrm{Et}$ dixit Dominus ad diabolum: imperet in te Cypran.

3 Dominus qui elegit Hierusalem ${ }^{3}$ [Cyprian.] Et Iesus erat indutus vestimenta sordida, et stabat ante faciem ipsius 4 angeli. "Et respondit et ait ad eos qui stabant ante faciem eius, dicens : auferte vestimenta sordida ab eo. Et dixit ad eum : ecce 5 abstuli iniquitates tuas. Et induite eum poderem, ${ }^{5} \mathrm{et}$ inponite cidarim mundam super caput eius

$8^{8}$. . . . . ecce ego adduco puerum meum,

9 ortus nomen ei est. ${ }^{\circ}$ Quoniam lapis quem dedi ante faciem Iesu super lapidem ipsum unum septem oculi sunt

IV.

Tyconius. $\quad 9^{\circ}$ Manus Zorobabel fundaverunt domum hanc et manus eius perficient eam

Il 7-9 Spec. ii II 8 Tert. Adv. Mare. iv 35

II $\mathrm{r}_{3}$ Tycon. Reg. Sept. III I Cypr. Testim. ii 13 III a Spec. ii III 3-5 Cypr. Testzm. ii 13 III 8, 9 Cypr. Testim. ii 16 IV 9 Tycon. Reg. Sept.

II. 8. qui tangit ad fin. com.] qui tetigerit vos ac si pupillam oculi mei tangat Tert

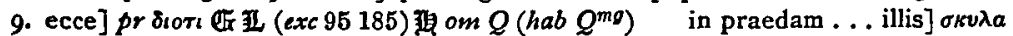

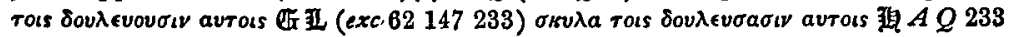

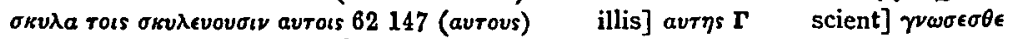

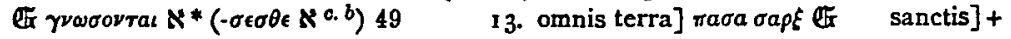
avrov ofr

III. r. illum] om of

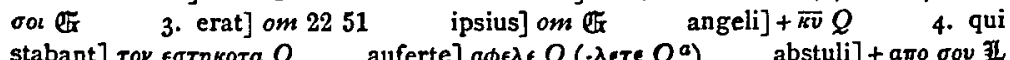

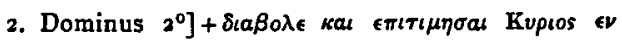

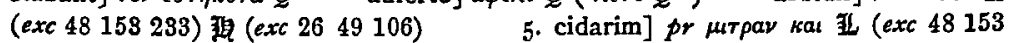

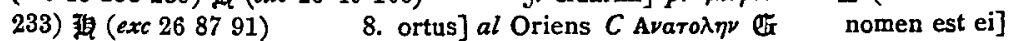

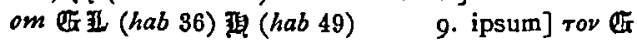

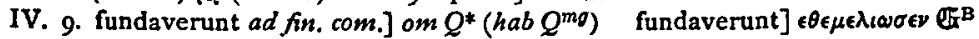

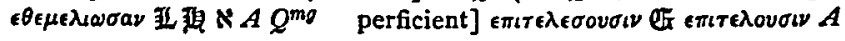


$14^{14}$

Illi duo filii opimitatis adsistunt Tertullian.

Domino universae terrae.

V. I' Et conversus adlevavi oculos meos et vidi et ecce falcem Speculum. 2 volantem $^{2}$. . . . . . statura cubitorum viginti et

3 latitudo cubitorum decem. ${ }^{3}$ Et dixit mihi: hoc est maledictum quod exiit ad maleficos qui sunt super faciem totius terrae; quia 4 omnis fur et periurus ex ea usque ad mortem punietur 4 .

Et proferam illam, dicit Dominus omnipotens, et introibit in domum furis et in domum periurantis in nomine meo in mendacio, et requiescet in media domo eius, et consummabit eum et materiam eius et lapidem eius

VII.

$9^{\circ} \mathrm{Haec}$ dicit Dominus omnipotens: iudicium iustum iudicate; et ro misericordiam et pietatem facite unusquisque ad fratrem suum, ${ }^{10}$ et viduam et orfanum et proselytum et pauperem per potentiam nolite obprimere et malitiam unusquisque fratris sui ne rememoremini in It cordibus vestris. ${ }^{11}$ [Luc. Cal.] Et dissuaserunt ne observarent et Luc. Cal.

I dederunt dorsum stultitiae et aures suas ${ }^{22}$ et cor suum statuerunt insuadibile ne oboedirent, degravaverunt ut non oboedirent legem meam .

$13^{13}$

exaudiam eos, dicit Dominus omnipotens.

VIII.

IV 14 Tert. Adv. Marc. iv 22

VII 9 , ro Spec. $x$ VII 9-13 Lucif. Cal. De sanct. Athan. i 37 VII ro Tert. Adv. Marc. iv I6

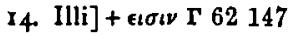

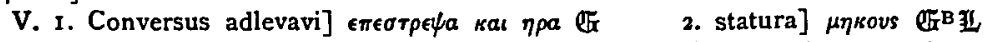

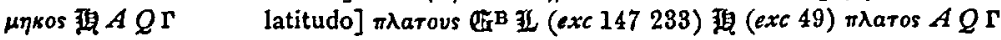

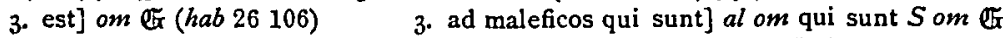

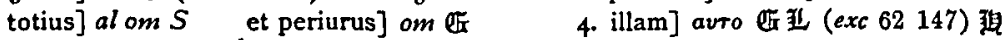

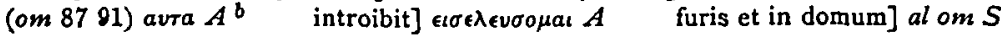

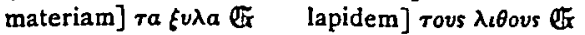

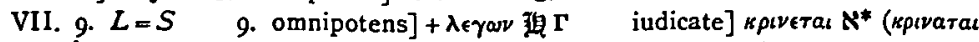
$\left(x^{c . a, c . b}\right.$ fratrem] $\pi \lambda \eta \sigma o v$ [L $\quad$ 10. per potentiam] om [fr obprimere] nocere $L$ malitiam ad fin. com.] malitiam unusquisque non reminiscatur fratris sui in corde suo $L$ ne unusquisque malitiae fratris sui meminerit sed nec proximi

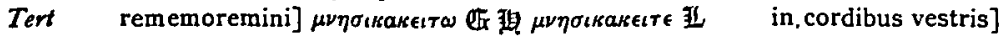
Tov $a \delta € \lambda \phi \circ v$ autov $Q$ II. dorsum] + avt $\omega \nu$ i[ et aures suas] + $\epsilon$ Bapvvav tov

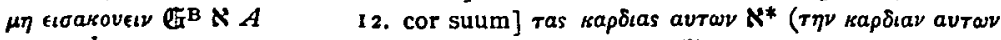
(x.a, c.b) degravaverunt ut non oboedurent] om fir 13. si] ovtows [f

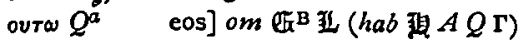


Cyprian.

${ }^{15}{ }^{15}$

16 quies (ci)tote; 10 haec sunt verba quae faciatis: unusquisque ad proximum suum et judicium pacificum et iustum

${ }_{17}$ iudicate in portis vestris, ${ }^{17}$ et unusquisque malitiam proximi tui nolite cogitare in cordibus vestris, et iusiurandum falsum nolite diligere, quoniam haec omnia odi, dicit Dominus omnipotens $19^{19}$.

IX.

- pacem et veritatem diligite

$9^{8}$ Dicite filiae Sion: Ecce rex tuus venit tibi, iustus et salvans, mitis sedens super asinum indomitum

$\mathrm{X}$.

II "Et transibunt per mare angustum et percutient in mari fluctus, et arefacient omnes altitudines fluminum, et confundetur omnis iniuria I2 Assyriorum, et sceptrum Aegypti auferetur. ${ }^{12} \mathrm{Et}$ confortabo eos in Domino Deo ipsorum, et in nomine eius gloriabuntur, dicit Dominus

XI.

Cyprian.

$16{ }^{18}$ Ecce ego suscito pastorem in terra qui quod aversum est non visitabit manducabit, et talos illorum torquebit .

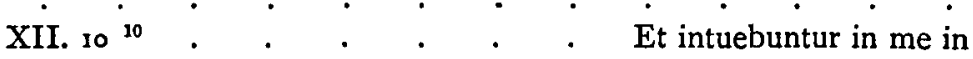
VIII ${ }^{1-17}$ Lucif. Cal. De sanct. Athan. i $37 \quad$ VIII 17 Tert. Adv. Marc. iv 16; Spec. xl VIII Ig Lucif. Cal. De sanct. Athan. i 37 IX 9 Cypr. Testim. ii $29 \mathrm{X}$ 11, 12 Cypr. Testim. ii. 6 X 12 Spec. ii XI 16 Cypr. Ad Novat. xiv XII 10 Cypr. Testim. ii $20 \quad$ Tert. De Resurrec. Cam. xxvi ; Adv. Marc. iii 7 ; Adv. Iud. iv

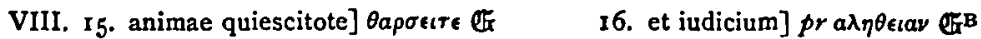

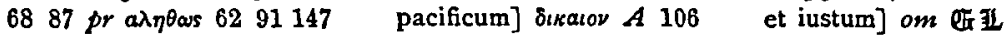

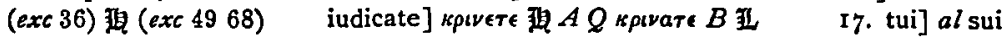
$L$ Tert avtov [4 223668 nolite cogitare] unusquisque ne recogitet $T$ ert iusiurandum] iuramentum $S$ quoniam] quia $S$ odi] odivi $S$ r. pacem et veritatem] bis scr $N$ veritatem] tr [fr

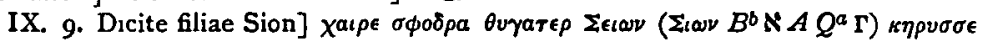

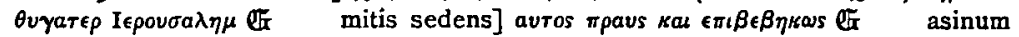

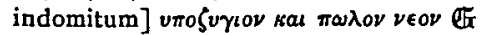

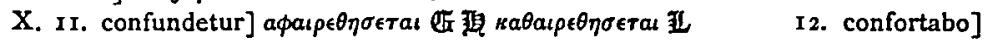
confirmabo $S$

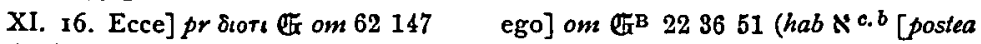

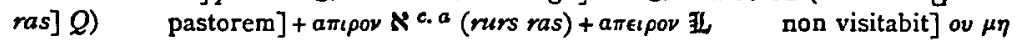

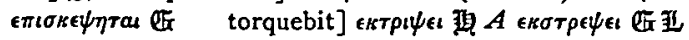

XII. 10. Videbunt enim eum qui confixerunt al Tunc et cognoscent eum qui compugerunt al Et tunc cognoscent eum quem pupugerunt Tert in me] + (?) ets 
quem transfixerunt

XIII.

$7^{7}$ Exurge romphaea in pastores oves, et superducam manum meam in pastores $9^{\circ}$ uritur argentum, et probabo illos sicut probatur aurum . . .

xiv.

12 in Hierusalem confidens, ${ }^{12}$ et haec erit strages qua caedet Dominus populos, quotquot militaverunt adversus Hierusalem: tabescent carnes eorum stantibus eis super pedes suos, et oculi eorum fluent

13 a foraminibus eorum, et lingua eorum tabescet in ore eorum. ${ }^{13} \mathrm{Et}$ erit in illa die alienatio magna super illos, et adprehendet unusquisque manum proximi sui, et implicabitur manus eius manui 14 proximi eius. ${ }^{14} \mathrm{Et}$ Iudas proeliabitur in Hierusalem, et colliget vires omnium populorum, aurum et argentum et vestem in multituI5 dinem nimis. ${ }^{15} \mathrm{Et}$ haec erit strages equorum et mulorum et camelorum et asinorum et omnium pecorum quae sunt in castris 16 illis, secundum stragem istam. ${ }^{18} \mathrm{Et}$ erit quicumque relicti fuerint ex omnibus gentibus venientibus super Hierusalem et ascendent quotquot annis adorare regem Dominum omnipotentem, celebrare diem festum scenopegiae

\section{MALACHI.}

I. $2^{2}$.

XIII 7 Tert, De Fuga in persec. xi Iacob dilexi, Cyprian. XIV $11-16$ Tycon. Reg. Quart. XIV I4 Tert. Adv. Marc. iii I3; Adv. Iud. ix Malachi. I 2, 3 Cypr. De Mont. Sin. et Sion. vi

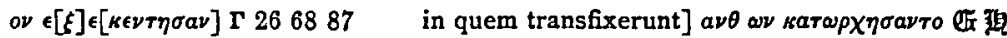

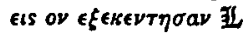

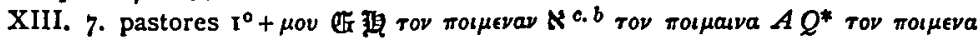

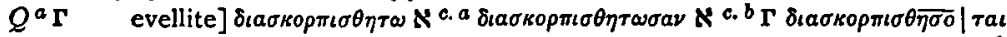

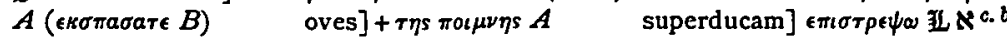

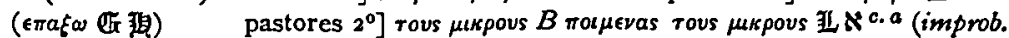

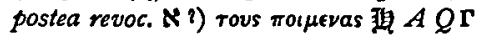

XIV. II. in] om [f $\quad I 1,12$. confidens .... adversus Hierusalem] om $\mathbb{N}^{*}$

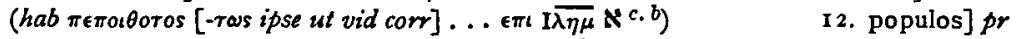

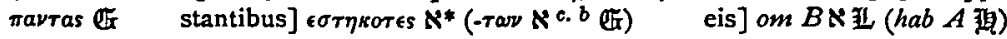
a] $\epsilon \kappa$ ff $a \pi 0 A$ I3. alienatio] + Kuptov ffr (exc 106) adprehendet] $\epsilon \pi i \lambda \eta \mu$ -

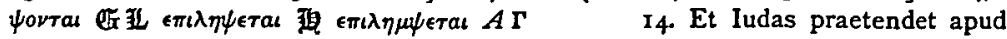
Hierusalem et congregabit omnem valentiam populorum per circuitum aurum et

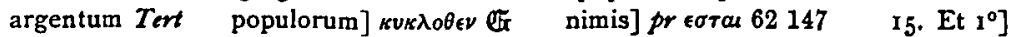

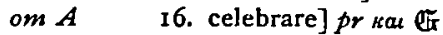

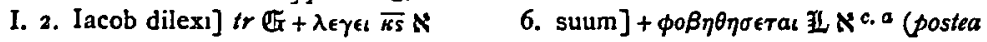




\section{$57^{6}$ THE JOURNAL OF THEOLOGICAL STUDIES}

Luc. Cal. $\quad 6{ }^{6}$ Filius honorificat patrem et servus dominum suum; et si.pater sum Coll. Carth. ego, ubi est honor meus? dicit Dominus omnipotens. [Coll. Carth.] Vos, o sacerdotes, qui profanatis nomen meum et dixistis, in quo pro7 fanavimus nomen tuum ? ${ }^{7}$ Et ponentes in altari meo panes pollutos; et dixistis, in quo polluimus illos? In eo ut diceretis, mensa Domini benedicta est et quae superponebantur annullastis . .

Cyprian.

Speculum.

Cyprian.

$$
\begin{aligned}
& \text { 10 }{ }^{\circ} . \\
& \text { circa vos, dicit Dominus, et sacrificium acceptum non habebo ex } \\
& \text { II manibus vestris; " quoniam a solis ortu et usque in occasu clarifica- } \\
& \text { tum est nomen meum apud gentes, in omni loco odores incensi } \\
& \text { offeruntur nomini meo et sacrificium mundum, quoniam magnum } \\
& \text { est nomen meum apud gentes, dicit Dominus, et sacrificium ac- } \\
& \text { ceptum non habebo ex manibus vestris. }
\end{aligned}
$$

I 6 Lucif. Cal. De sanct. Athan. i 37 I 6, 7 Coll. Carth. Gesta cclviii ; Spec. xlvi I 10 Spec. xlvi I Io, 11 Cypr. Testim. i 16; Tert. $A d v$. Marc. iii 22; Adv. Iud. v; Coll. Carth. Gesta lv I I4 Spec. lix; Cypr. Testrm. ii 29

ras) o] om $S$ [ [ profanatis] fallitis $S$ in quo] in qua re $S$ profanavimus] fefellimus $S$ 7. Et] om $S[$ [ ponentes] offerentes $S$ in altari meo] ad altarium meum $S$ et dixistis] in mg. ras asterisc $B$ ? bene-

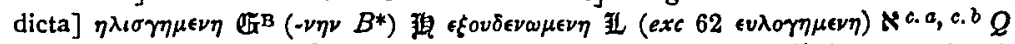
et quae superponebantur] in mg. ras asterisc $B^{2}$ kal $\tau a \in \pi i \theta \in \nu a N^{*}\left(-\theta \in \mu \in \nu a N^{1}, c_{0} a\right)$

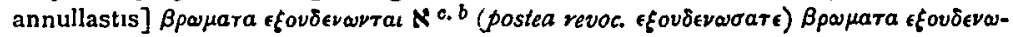
$\mu \in \nu a$ 㼭 $A Q \quad$ Io. circa vos] in vobis $C c$ om $S \in \nu \nu \mu \nu$ [f Dominus] +omnipotens $C c S+$ navrokparop [f et sacrificium] sacrificia Tent acceptum] om

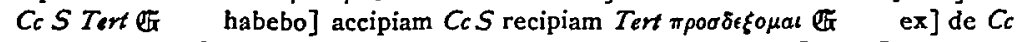
11. a solis ortu] ab ortu solis $C_{c}$ ab oriente sole Tert et $\mathrm{I}^{\circ}$ ] om $C_{c}$ Tert $A Q$

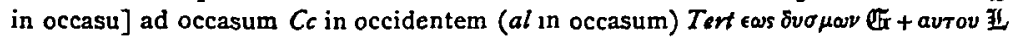
clarificatum] glorificatum (al clarificatum) $T e r t \quad$ apud gentes $1^{\circ}$ ] in gentibus $C_{c}$ in nationibus (al in omnibus gentibus) Tert +et $C_{c}$ Tert $\left(\epsilon \theta \nu \in \sigma t\right.$ bis $\left.Q^{a}\right)$ in $2^{\circ}$ ] $\mathrm{pr}$ et [f odores incensi offeruntur] incensum offertur $C c$ offeruntur (al offeren-

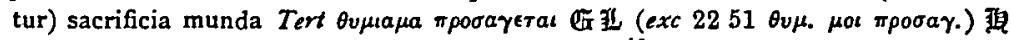

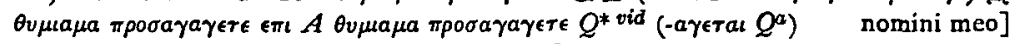
om 2251 quoniam $2^{\circ} \ldots$ apud gentes $2^{\circ}$ ] om Tert apud gentes $2^{\circ}$ ] in gentibus $C_{c}$ Dominus] + omnipotens $C_{c}+\pi a \nu$ rokpatop of et sacrificium $2^{\circ}-a d$

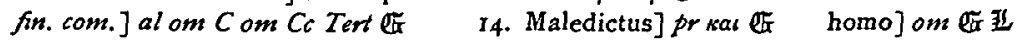
(exc 36) 理 (hab Compl) erat $2^{\circ}$ ] +auro $A 26$ eius $1^{\circ}$ ] om $A$ immolat]

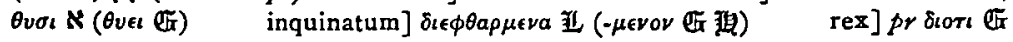

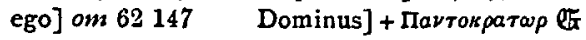


II. I, $2{ }^{1}$ Et nunc praeceptum hoc ad vos est, o sacerdotes. ${ }^{2} \mathrm{Si}$ non Cyprian. audieritis et si non posueritis in corde vestro ut detis. honorem nomini meo, dicit Dominus omnipotens, immittam in vos maledictionem, et maledicam benedictionem vestram

$5^{5}$ Testamentum meum fuit cum vita et pace, et dedi illi timorem ut $6{ }^{6}$ timeret me a facie nominis mei proficisci illum. ${ }^{\circ}$ Lex veritatis in ore eius, et iniustitia non est inventa in labiis eius, in pace linguae 7 corrigens ambulavit nobiscum, et multos avertit ab iniustitia. ${ }^{7}$ Quoniam labia sacerdotis servabunt scientiam, et legem exquirent $a b$ ore eius, quoniam angelus Domini Omnipotentis est

Io ${ }^{10}$ Nonne Deus unus condidit nos? Nonne pater unus est omnium nostrum? quid utique dereliquistis unusquisque fratrem suum . . II ${ }^{11}$ Derelictus est Iuda et abominatio facta est in Israel et in Hierusalem, quoniam profanavit Iudas sancta Domini, in quibus dilexit, 12 et affectavit deos alienos. ${ }^{12}$ Exterminabit Dominus hominem qui facit haec, et humilis in tabernaculis Iacob

${ }_{17}{ }^{17}$ Exacerbatis Deum in verbis vestris, et dixistis, in qua re exacer- Luc. Cal. bavimus eum? In eo quod dicatis : omnis qui facit malum bonum est coram Deo, et in ipsis benedicet. Et ubi est Deus iustitiae? III. $I^{1} \quad . \quad . \quad . \quad . \quad . \quad . \quad . \quad . \quad$ Et subito venit Speculum. in templo suo Dominus quem vos quaeritis, et angelus testamenti quem vos vultis;

$3^{3} \mathrm{Et}$ sedit conflans et expurgans sicut aurum et argentum et emun- Cyprian.

II I, 2 Cypr. Ep. lix 13; lxxiv 8 II 5 Cypr. Testim. iii 20 II 5-7 Cypr. Testim. ii 5 II Io Cypr. Testim. iii 3 II II, I 2 Cypr. Testim. i I II I7 Lucif. Cal. De sanct. Athan. 138 III I Spec. ii III 3 Cypr. Testim. iii 57

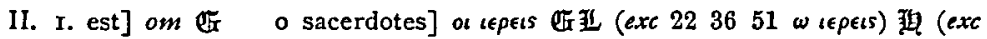

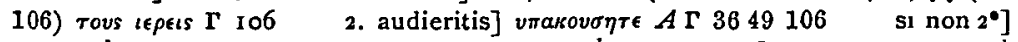

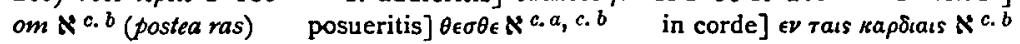
(postea repos. eis $\tau \eta^{\nu}$ rapótav) omnipotens] al om $C$ immittam] al inmittam $C$ $\epsilon \xi a \pi \operatorname{\sigma o\sigma } \epsilon \lambda \lambda \omega N\left(-\sigma \tau \in \lambda \omega \aleph^{c a, c . b}\right)$ 5. meum] al om $C$ (om 311) vita] pravrov

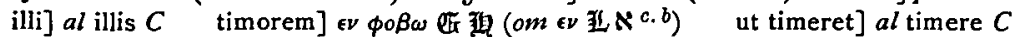
me] om $B$ (hab $B^{a b}(v i d)$ a facie] pr wac [fi proficisci illum] al om $C$ 6. in ore] $p r \eta v$ [er linguae] al om $C$ om [4 nobiscum] $\mu \epsilon \tau \in \mu o v$ [ir 7 . Om-

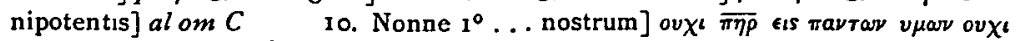

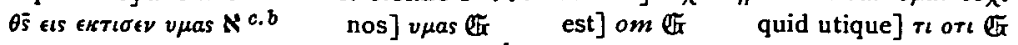

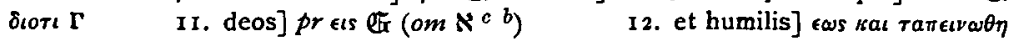

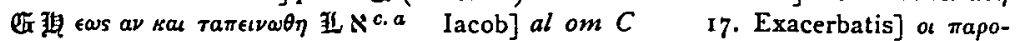

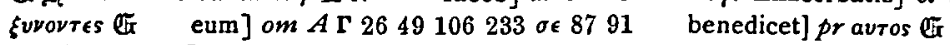

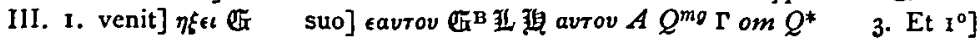
vOL. v. 


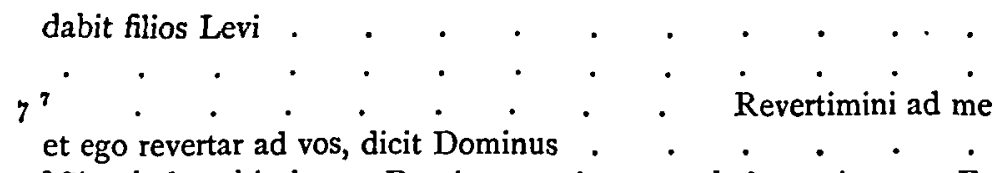

Speculum. $8^{8} \mathrm{Si}$ subplantabit homo Dominum quia vos subplantastis me. Et dixistis, in quo subplantavimus te? Quia decimae meae et primitiae 9 vobiscum sunt. 'Et dissimulantes vos dissimulastis, et vos me ro subplantastis, $\ldots{ }^{10}$ et intulistis vos pignera in thensauris vestris, et

erit rapina in domibus vestris

Luc. Cal. $\quad{ }_{13}^{13}$ Gravastis adversum me consilia vestra dicit Dominus omnipotens, $\mathbf{1}_{4}$ et dixistis, in qua re detraximus de te ? ${ }^{14}$ quia dixistis, vanus est omnis qui servit Deo, et quid amplius quod custodivimus praecepta illius, et ambulavimus deprecantes ante conspectu Domini omnipotentis?

${ }_{15}{ }^{15} \mathrm{Et}$ nunc nos magnificamus alienos, et renovantur omnes facientes I6 iniqua, et restiterunt Deo, et salvati sunt. ${ }^{16} \mathrm{Haec}$ detraxerunt qui timent Deum unusquisque ad proximum suum, et intendit Dominus et exaudivit, et scripsit librum memorialem coram se timentibus ${ }_{17}$ Dominum et metuentibus nomen suum. ${ }^{17}$ Et erit mihi, dicit Dominus omnipotens, in die qua ego facio, in possessione, et eligam eos quemadmodum eligit homo filium suum bene servientem sibi. ${ }_{18}{ }^{18} \mathrm{Et}$ conversi videbitis quantum sit inter iustum et iniustum, inter servientem Deo et non servientem.

III 7 Cypr. Ad Vig. i ; De bono pat. iv - III 8-10 Spec. lix III 13-18 Lucif. Cal. De sanct. Athan. i 38

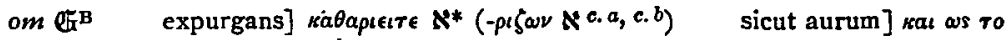
$\chi \rho v \sigma i o v$ obel adpinx $B^{a}$ (non b) mo aurum et argentum] tr [4 argentum] pross

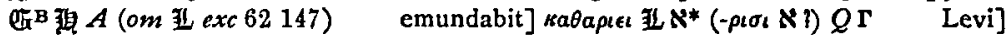

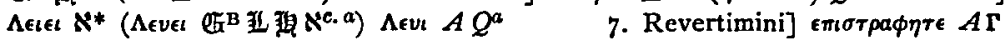
49106 ego] om [r et ego revertar ad vos] al om $C$ Dominus] + חavro-

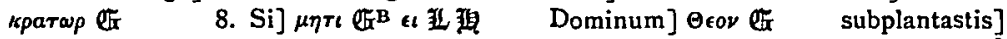

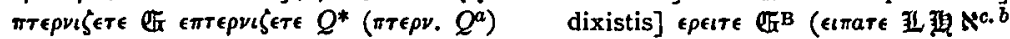

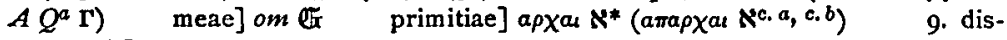

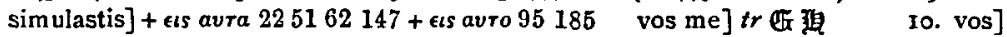

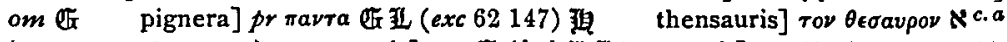

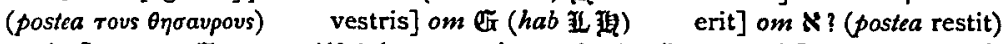
rapina] + avrov [4 + avtav $\aleph^{c . a}$ (postea ras) in domibus vestris] $\epsilon \nu$ To otrow avrou

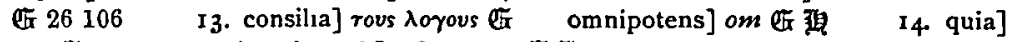

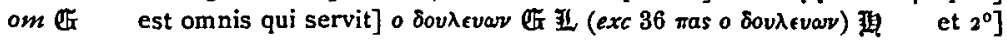

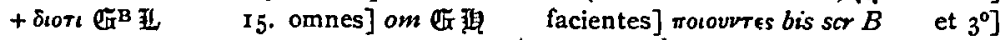

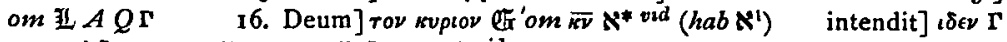

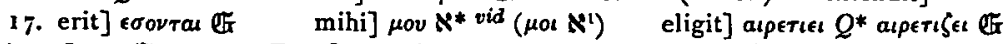
bene] om [fr 18. Et $x^{\circ}$ ] om $\aleph^{*}\left(h a b \aleph^{c . a}\right)$ quantum sit] om [fr iniustum]

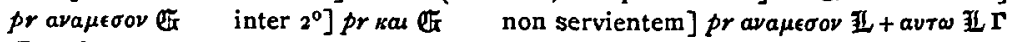
Compl 
IV. ${ }^{1}$ Ecce dies Domini venit ardens velut clibanus, eruntque Cypran. omnes alienigenae et omnes iniqui stipula, et succendet illos adveniens dies, dicit Dominus [Lucif. Cal.] et non derelinquetur ex eis Luc. Cal. 2 radix nec vitis. ${ }^{2}$ Vobis autem timentibus meum nomen orietur sol Cypran. iustitiae et sanitas in alis eius

'Et ecce mittam vobis Helian Thesbiten . . Tertullant

IV I Cypr. Testim. ii 28 ; Ad Demet. 22; Lucif. Cal. De sanct. Athan. i 38 IV 2 Cypr. Ad Vig. 6 ; De Pascha Computus I9; Tycon. Reg. Sept. $1{ }_{4}$; Tert. De Anima v

IV. I. Quia ecce dies venit Domini ardens sicut clibanus et uret eos et erunt omnes alienigenae et omnes qui faciunt scelera ut sarmenta et succendet eos dies Domini quae venit dicit Dominus omnipotens $L$

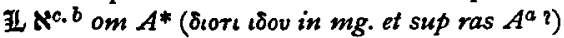

Ecce] pr $\delta t o \tau t$ 的画 prort Domini] om [F 1 il eruntque]

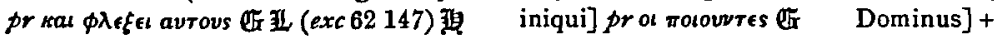

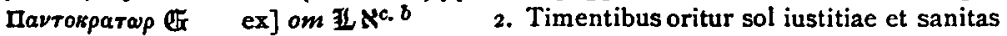

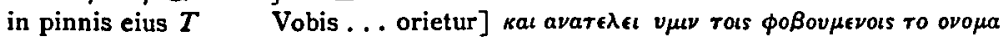
mov [r timentibus] al qui timetis $C$ meum nomen] al nomen Domini $C$ orietur] al +vobis $C$ sanitas] al curatio $C$ alis] al pinnis $C$ eius] avtav

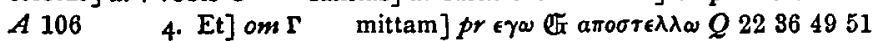

W. O. E. Oesterley.

\section{NOTES ON THE DIDACHE.}

\section{I.}

\section{On Baptism by AfFusion.}

WHERE and when did Baptism by Affusion come to be regarded as perfectly adequate and legitimate? We need not pursue the question beyond the first five centuries.

I. Tertullian de Cor. Mil. 3 says 'ter mergitamur'. In several places he uses the word 'tinguo', which means 'to dip' (Virg. Georg. i 246 'Arctos Oceani metuentes aequore tingui'); or to dip cloth in vats and so 'to dye'. The word does not appear to be used of bathing. Dipping which imparts a colour or character seems to be its common sense, and hence it came to be used of Baptism.

2. In the third century Cornelius, Bishop of Rome, says that the whole of the clergy and many of the laity of that church objected to

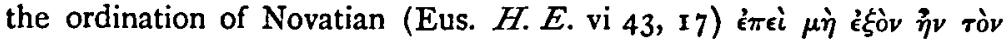

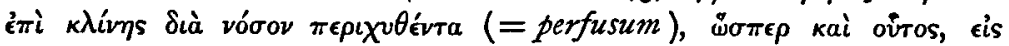

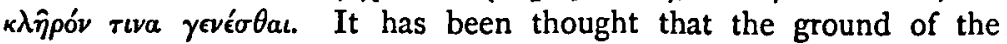

